\title{
Proposal of a new double hardening elasto-plastic constitutive model of soil skeleton based on integration of associated and non-associated flow rules
}

\author{
Shotaro Yamada ${ }^{\text {i) }}$ and Toshihiro Noda ${ }^{\text {ii) }}$
}

i) Associate Professor, Department of Civil Engineering, Nagoya University, Chikusa, Nagoya, 464-8603, Japan. ii) Professor, Disaster Mitigation Research Center, Nagoya University, Chikusa, Nagoya, 464-8603, Japan.

\begin{abstract}
A sophisticated elasto-plastic constitutive model of soil skeleton was newly proposed. The proposed model is built on the basis of the SYS Cam-clay model in which soil skeleton structure concept is included. A new loading surface was introduced into the SYS Cam-clay model based on associated flow rule, and a Drucker-Prager model based on non-associated flow rule was mounted on the model. A new framework of double hardening elasto-plasticity was developed for integration of two different types of elasto-plastic models. Although there are some existing constitutive models called "double hardening model" other than the model proposed in this paper, these are the ones produced by simply connecting yield surfaces or by separately utilizing loading surfaces for isotropic and shear components. The proposed model is clearly distinguished from the existing double hardening models in a sense that two types of elasto-plastic models which should exist independently can work dependently showing their combined loading condition. In this paper, basic formulation and essentials of the proposed model were firstly shown. In addition, basic responses of typical sand and clay described by the proposed model were demonstrated compared with experimental results. Especially, some hollow cylinder torsional tests for sand specimen controlled by switching drained condition during shear were focused on, and it was demonstrated that the soil behaviors seen in those tests can be described not by Cam-clay type models but by the proposed model.
\end{abstract}

Keywords: elasto-plastic, double hardening, combined loading

\section{INTRODUCTION}

This paper proposes a new framework called the "double hardening elasto-plastic constitutive equation" for the elasto-plastic constitutive equation of soil structure. A number of double hardening models other than the one proposed here do exist. However, these are models such as the Cap Model (Drucker et al., 1957), which utilizes linking up of yield functions, or models (Banerjee et al., 1992) that prepare separate loading surfaces for the isotropic and shear components. The model proposed here is distinguished from other models by the fact that it is configured using two models that could exist independently but take on mutually compatible states under conditions of combined loads. The authors endeavor, within the limited space available, to explain the basic structure of the proposed model and also to describe the basic responses.

\section{OUTLINE OF THE MODEL}

The SYS Cam-clay model (Asaoka et al., 2002), which describes the work of the soil skeleton structure, is used as the base of the model proposed here. In this model, a "midloading surface" is added anew between the superloading (Asaoka et al., 2000) and subloading (Hashiguchi, 1989) surfaces as shown in Fig. 1. By configuring the model so that the subloading surface approaches the midloading surface rapidly in response to marginal plastic deformation, the midloading surface is made into a region inside which it becomes difficult for plastic deformation to occur. This model is then combined with a second model (hereafter referred to as the DP model), in which the same elliptical plastic potential surface as that in the SYS Cam-clay model is adopted for the conical yield surface of the Drucker-Prager model (Drucker and Prager, 1952) shown in Fig. 2. The normal yield surface of the second model is assumed to be non-hardening, and on its inward side, we establish midloading and subloading surfaces. The two subloading surfaces exhibit smooth elasto-plastic behavior while sharing the current stress at all times. The fundamentals of formulating the double hardening elasto-plastic constitutive equation by 
combining these two models are described below.

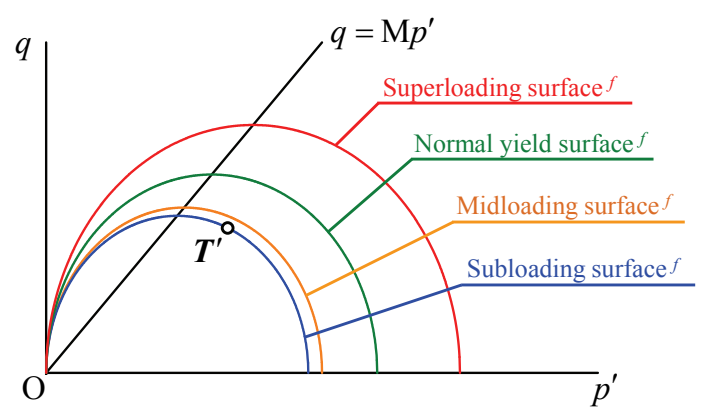

$$
\begin{aligned}
& R^{* f}=\frac{\text { Normal yield surface }^{f}}{\text { Superloading surface }^{f}} \\
& R_{1}^{f}=\frac{\text { Midloading surface }^{f}}{\text { Superloading surface }^{f}} \\
& R_{1}^{f}=\frac{\text { Subloading surface }^{f}}{\text { Midloading surface }^{f}}
\end{aligned}
$$

Fig.1 Loading surfaces of the SYS Cam-clay model

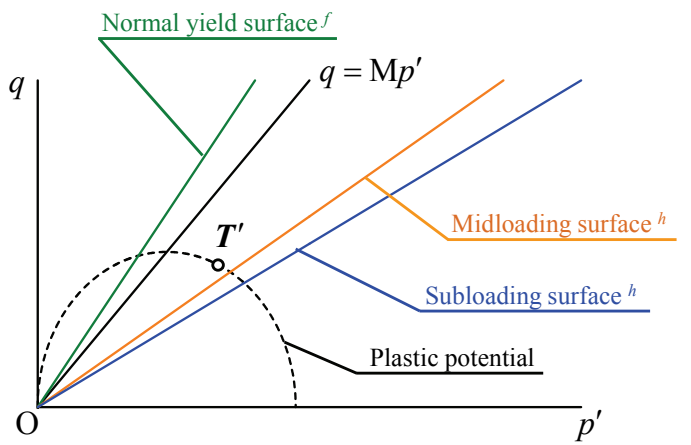

$$
\begin{aligned}
& R_{1}^{h}=\frac{\text { Midloading surface }^{h}}{\text { Normal yield surface }^{h}} \\
& R_{2}^{h}=\frac{\text { Subloading surface }^{h}}{\text { Midloading surface }^{h}}
\end{aligned}
$$

Fig.2 Loading surfaces of the DP model

\section{FORMULATION OF THE ELASTO-PLASTIC CONSTITUTIVE EQUATION}

\subsection{Additive decomposition of stretching and the} four states of loading/unloading

The stretching $\boldsymbol{D}$ (tension: positive) is decomposed additively into the elastic component $\boldsymbol{D}^{e}$ and the plastic component $\boldsymbol{D}^{p}$. The plastic component $\boldsymbol{D}^{p}$ is again decomposed additively into two components, $\boldsymbol{D}^{p f}$ and $\boldsymbol{D}^{p h}$.

$$
\begin{gathered}
\boldsymbol{D}=\boldsymbol{D}^{e}+\boldsymbol{D}^{p}=\boldsymbol{D}^{e}+\boldsymbol{D}^{p f}+\boldsymbol{D}^{p h} \\
\left(\boldsymbol{D}^{p}=\boldsymbol{D}^{p f}+\boldsymbol{D}^{p h}\right)
\end{gathered}
$$

The states of loading/unloading are classified into the following four types based on the combinations of the states of occurrence/nonoccurrence of the above two plastic components.
[1] $\boldsymbol{D}^{p f} \neq \mathbf{0}, \boldsymbol{D}^{p h} \neq \mathbf{0}$ : combined loading

[2] $\boldsymbol{D}^{p f} \neq \mathbf{0}, \boldsymbol{D}^{p h}=\mathbf{0}$ : singular $f$ component loading

[3] $\boldsymbol{D}^{p f}=\mathbf{0}, \boldsymbol{D}^{p h} \neq \mathbf{0}$ : singular $h$ component loading

[4] $\boldsymbol{D}^{p f}=\mathbf{0}, \boldsymbol{D}^{p h}=\mathbf{0}$ : fully unloaded

\subsection{Elastic constitutive equation}

The following equation, which is the rate-type form of the hyperelastic constitutive equation proposed by Einav and Puzrin (2004), is adopted as the elastic constitutive equation.

$$
\stackrel{\circ}{\boldsymbol{T}^{\prime}}=p^{\prime}\left(2 \bar{G} \boldsymbol{D}_{s}^{e}+\frac{1}{\tilde{\gamma}} \frac{\boldsymbol{T}^{\prime}}{p^{\prime}} \cdot \boldsymbol{D}^{e} \frac{\boldsymbol{T}^{\prime}}{p^{\prime}}\right)
$$

In the above equation, $\stackrel{\boldsymbol{T}}{\prime}^{\prime}$ is the Green-Naghdi rate (Green and Naghdi, 1965) of the Cauchy stress $\boldsymbol{T}^{\prime}$ (tension: positive), $\boldsymbol{D}_{s}^{e}$ is the deviator component of $\boldsymbol{D}^{e}$, and $\bar{G}$ and $\tilde{\gamma}$ are material constants.

\subsection{Yield functions}

The two types of yield functions shown below are adopted.

Yield function $F$ :

$$
\begin{aligned}
& F=f\left(p^{\prime}, \eta\right)+(\widetilde{\rho}-\tilde{\gamma}) \ln \frac{R^{* f}}{R_{1}^{f} R_{2}^{f}}-\varepsilon_{v}^{p} \\
& =(\widetilde{\rho}-\tilde{\gamma}) \ln \frac{p^{\prime}}{P_{0}^{\prime}} \frac{\mathrm{M}^{2}+\eta^{2}}{\mathrm{M}^{2}}+(\widetilde{\rho}-\tilde{\gamma}) \ln \frac{R^{* f}}{R_{1}^{f} R_{2}^{f}}-\varepsilon_{v}^{p}=0
\end{aligned}
$$

Yield function $H$ :

$$
\begin{aligned}
H & =h(\eta)-\mu R_{1}^{h} R_{2}^{h} \\
& =\eta-\mu R_{1}^{h} R_{2}^{h}=0
\end{aligned}
$$

Yield function $F$ expresses the yield function of the SYS Cam-clay model, and yield function $H$ expresses that of the DP model. M, $\tilde{\rho}, \tilde{\gamma}$, and $\mu$ are material constants. $R^{* f}, R_{1}^{f}, R_{2}^{f}, R_{1}^{h}$, and $R_{2}^{h}$ express the homothetic ratio of each loading surface as shown within Figs. 1 and 2. $\varepsilon_{v}^{p}$ is the logarithmic volumetric strain, and $P_{0}^{\prime}$ denotes the position of the foot of the normal yield surface when $\varepsilon_{v}^{p}=0 . f\left(p^{\prime}, \eta\right)$ and $h(\eta)$ indicate the subloading surfaces that will be referred to as subloading surface ${ }^{f}$ and subloading surface $^{h}$, respectively. It should be noted that anisotropy is not taken into account because this paper places emphasis on explaining the basic structure of the proposed model.

\subsection{Flow rules}

Both plastic stretching components are defined by the flow rule, which makes the subloading surface ${ }^{f}$ a plastic potential surface. 


$$
\begin{aligned}
& \boldsymbol{D}^{p f}=\Lambda^{f} \frac{\partial f}{\partial \boldsymbol{T}^{\prime}} \quad\left(\Lambda^{f}>0\right) \\
& \boldsymbol{D}^{p h}=\Lambda^{h} \frac{\partial f}{\partial \boldsymbol{T}^{\prime}} \quad\left(\Lambda^{h}>0\right)
\end{aligned}
$$

$\Lambda^{f}$ and $\Lambda^{h}$ in the above equations are plastic multipliers. It follows that the associated flow rule has been applied for the SYS Cam-clay model, whereas the non-associated flow rule has been applied for the DP model.

\subsection{Evolution rule for internal state variables}

Although the specific form has been omitted due to space restrictions, each term is assigned a property that makes the homothetic ratio of each surface increase monotonously towards 1 along with the formation of $\boldsymbol{D}^{p f}, \boldsymbol{D}^{p h}$, etc.

\subsection{Plastic multipliers}

The two plastic multipliers $\Lambda^{f}$ and $\Lambda^{h}$ of Equation (5) are determined from the consistency conditions corresponding to the two subloading surfaces. The consistency conditions are solved singly in the case of singular loading and simultaneously in the case of combined loading. It is possible to express perfunctorily the plastic multipliers corresponding to the loading conditions shown in section $\mathbf{3 . 1}$ by the following equations.

[1] $\Lambda^{f}=\frac{\left(d \frac{\partial f}{\partial \boldsymbol{T}^{\prime}}-b \frac{\partial h}{\partial \boldsymbol{T}^{\prime}}\right) \cdot \boldsymbol{E D}}{a d-b c}$,

$$
\Lambda^{h}=\frac{\left(a \frac{\partial h}{\partial \boldsymbol{T}^{\prime}}-c \frac{\partial f}{\partial \boldsymbol{T}^{\prime}}\right) \cdot \boldsymbol{E D}}{a d-b c}
$$

[2] $\Lambda^{f}=\frac{\frac{\partial f}{\partial \boldsymbol{T}^{\prime}} \cdot \boldsymbol{E D}}{a}$,

[3] $\Lambda^{h}=\frac{\frac{\partial h}{\partial \boldsymbol{T}^{\prime}} \cdot \boldsymbol{E D}}{d}$

In the above equations, $\boldsymbol{E}$ is the elastic modulus tensor, and $a, b, c$, and $d$ are determined when the plastic multipliers are derived from the consistency conditions. As shown in Equations (6b) and (6c), a and $d$ are the denominators of the plastic multipliers at the time of singular loading. $b$ and $c$ have forms similar to those of $a$ and $d$, respectively.

In the condition of combined loading, the plastic stretching is expressed by the following equation.

$$
\boldsymbol{D}^{p}=\Lambda \frac{\partial f}{\partial \boldsymbol{T}^{\prime}},
$$

$$
\Lambda=\frac{\left\{(d-c) \frac{\partial f}{\partial \boldsymbol{T}^{\prime}}+(a-b) \frac{\partial h}{\partial \boldsymbol{T}^{\prime}}\right\} \cdot \boldsymbol{E D}}{a d-b c}
$$

\subsection{Elasto-plastic constitutive equation}

Based on the above relationship, the elasto-plastic constitutive equation is expressed as shown below.

$$
\stackrel{\circ}{\boldsymbol{T}^{\prime}}=\boldsymbol{E} \boldsymbol{D}-\Lambda \boldsymbol{E} \frac{\partial f}{\partial \boldsymbol{T}^{\prime}}
$$

There are matters other than the above that need to be explained. The loading criterion is one of them. After assigning the evolution rule of the internal state variables so as to satisfy the restrictions that need to be imposed on $a, b, c$, and $d$ and then assigning the loading criteria suitably, smooth responses are shown even when the loading states change. Another important factor is definition of the evolution of the internal state variables during unloading or singular loading in a rational manner. Because of the limited space available, we reserve the explanation regarding these points for another occasion and describe below only the basic responses to the model proposed here.

\section{SIMULATION OF THE BEHAVIOR OF SAND}

\subsection{Monotonic drained/undrained shear behavior}

Figures 3 and 4 illustrate the monotonic drained and undrained shear behaviors, respectively, of a soil assumed to be Toyoura sand. In both cases, the calculations were performed to simulate the behavior of specimens with relative densities of between 40 and $80 \%$ subjected to torsional shear under a constant vertical load, internal pressure, and external pressure. Consistently the same material constants were used for all tests. As for the initial conditions, the same initial values were assigned for any given value of specimen density in both the drained and undrained tests. The proposed model captured well the characteristics of the behavior of the sand under drained and undrained shear. Note that in these calculations the initial value of $R^{*} f$ was 1 , which means that the calculations have been performed under the condition of superloading surface $f$ being coincident with the normal yielding surface $f^{f}$ In the SYS Cam-clay model, the behavior of effective stress paths of loose sands or medium dense sands moving toward the origin has been simulated until now by considering it to be softening behavior caused by the degradation of structure (in the case of this model, the contraction behavior of the subloading surface $f$ accompanied by the formation of $\boldsymbol{D}^{p f}$ ). In the proposed model, this behavior is simulated by considering it to be a phenomenon caused by the fact that the looser the state of the specimen is, the slower the hardening behavior of the DP model is (expansion behavior of the subloading surface ${ }^{h}$ due to the formation of $\boldsymbol{D}^{p h}$ ). 

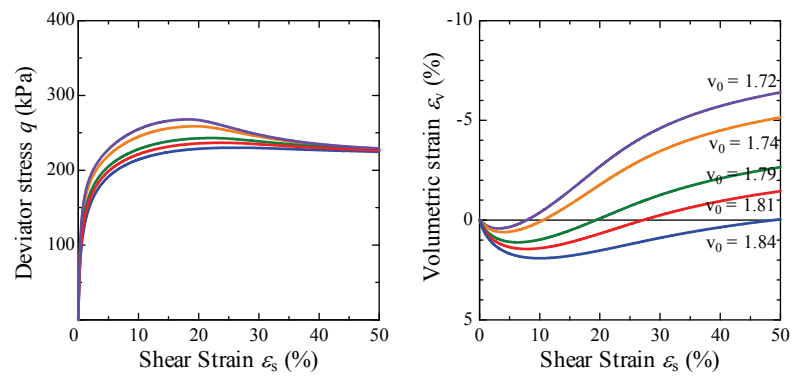

Fig.3 Drained shear responses of the double hardening elasto-plastic constitutive model
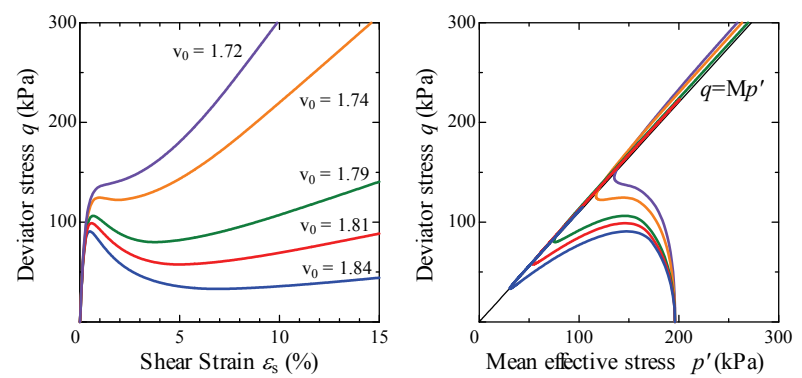

Fig.4 Undrained shear responses of the double hardening elasto-plastic constitutive model

\subsection{Drainage condition switching shear tests and their simulation}

Figure 5 depicts the results of drainage condition switching tests performed for Toyoura sand. The following four shear tests were performed: (1) drained shear test (CD test), (2) undrained shear test (CU test), (3) switching shear test from the drained condition to an undrained condition (CDU test), and (4) switching shear test from the undrained condition to a drained condition (CUD test). The tests were conducted using hollow cylinder torsional shear apparatus. Specimens were prepared using Toyoura sand by the aerial deposition method, and torsional shear was applied with the internal pressure, external pressure, and vertical load being kept constant. The targeted relative density of the specimens at the time of commencement of shearing was $D_{\mathrm{r}}=45 \%$. It can be observed that switching to the undrained condition while drained shearing was taking place resulted in a rapid decrease in the shear stress $q$. In contrast, switching to the drained condition while undrained shearing was taking place makes the shear stress $q$ increase from its decreasing trend before switching.

The results of simulation of the above tests using the proposed model are illustrated in Fig. 6. In the four calculations, only the drainage conditions differ, and all material constants and initial values are the same. The proposed model simulated the results of all tests with good accuracy. In the case of the proposed double hardening elasto-plastic constitutive equation, the loading transitions automatically from combined loading to singular loading by the $h$ component when the drainage condition is switched from drained shear to undrained shear. Conversely, when the drainage condition is switched from undrained shear to drained shear, the loading transitions automatically from singular loading by the $h$ component at the time of switching to combined loading. The above simulation became possible only because the phenomenon of the effective stress paths of loose sands or medium dense sands moving toward the origin was simulated not as the softening behavior of the SYS Cam-clay model but as the hardening behavior of the DP model.
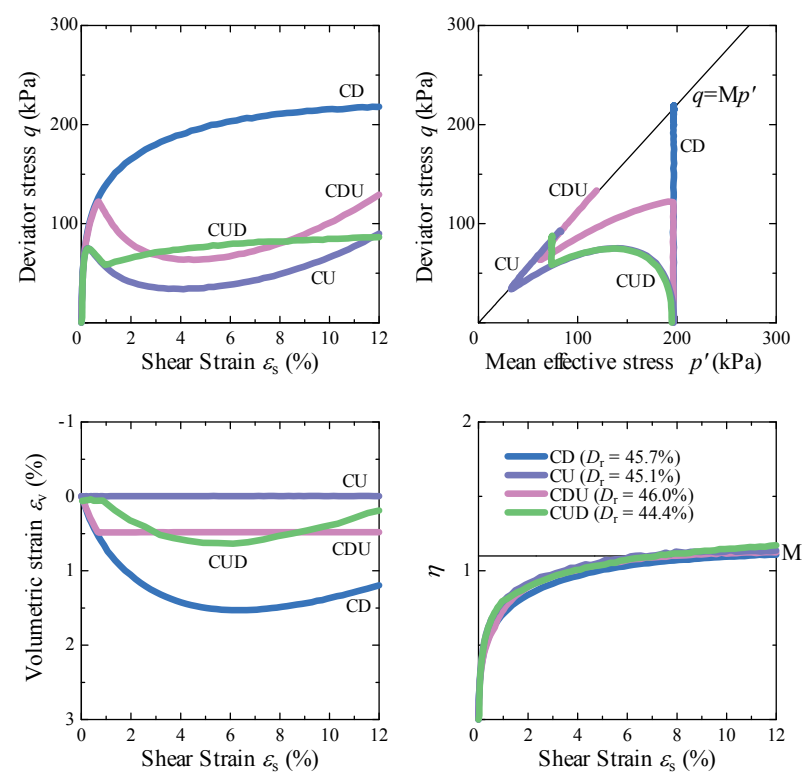

Fig.5 Drained condition switching shear test results Toyoura sand (experiment)
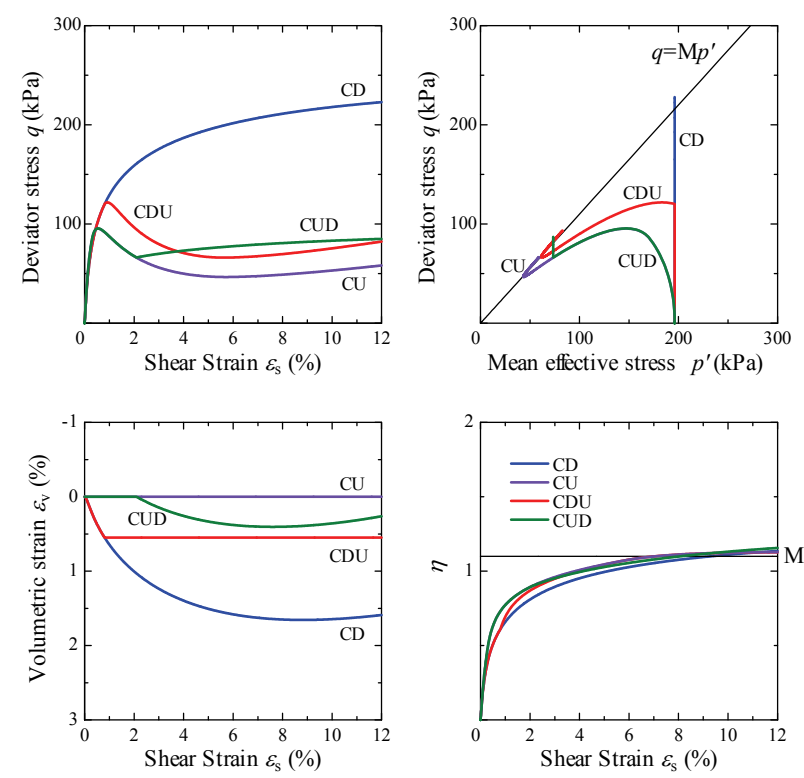

Fig.6 Reproduction of drained condition switching shear test results by the double hardening elasto-plastic model (analysis) 


\section{SIMULATION OF THE BEHAVIOR OF CLAY}

It is shown below that the proposed model is capable of simulating the behavior of not only sand but also that of clay. Figure 7 illustrates the results of monotonic undrained shear tests on undisturbed alluvial clay specimens sampled from Urayasu, and Fig. 8 illustrates the simulation of the results of these tests using the proposed model. The simulation, which was performed using a single set of material constants and initial values, included calculation of the stage of consolidation. It can be affirmed that the experimental results were replicated well by the proposed model. This type of behavior, which is obtained by configuring the midloading surface $^{h}$ to approach the normal yielding surface ${ }^{h}$ rapidly, can be understood by referring to Equation (7). First, configuring the midloading surface ${ }^{h}$ to rapidly approach the normal yielding surface ${ }^{h}$ makes $d$ in Equation (7) become more predominant than $a, b$, or $c$. As a result of this, the plastic multiplier at the time of combined loading becomes the same as the plastic multiplier at the time of singular $f$ component loading. In other words, under such conditions, the proposed model becomes the same as the SYS Cam-clay model. The behavior illustrated in Fig. 8 was obtained because the superloading surface and its evolution rule play the role, in the same manner as before, of describing the bulkiness and degradation of naturally deposited clay, as seen from remolded clay.
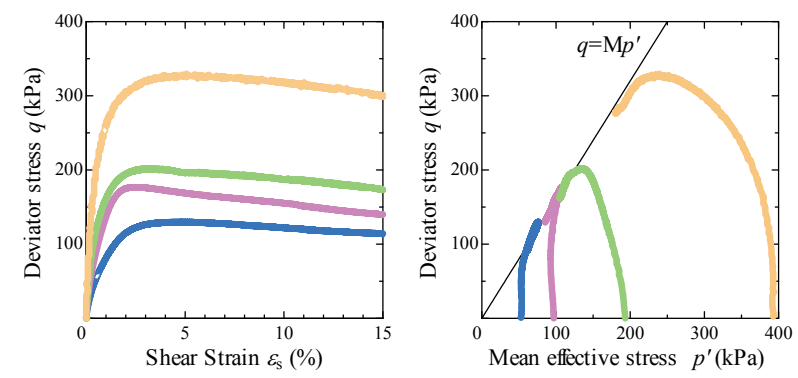

Fig.7 Undrained shear behaviors of Urayasu alluvial clay (experiment)
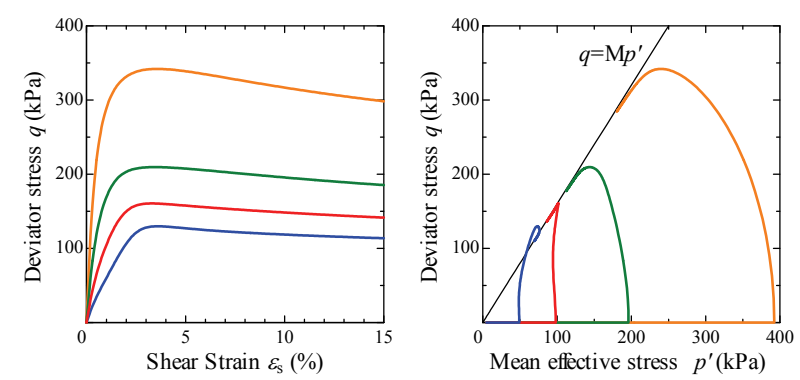

Fig.8 Reproduction of undrained shear behaviors of Urayasu alluvial clay by the double hardening elasto-plastic model (analysis)

\section{CONCLUSIONS}

The framework of a double hardening elasto-plastic constitutive equation was described here. Although only isotropic hardening was taken into consideration so that readers obtain an understanding of the basic structure of the model proposed in this paper, the existence of induced anisotropy too is important for simulating cyclic mobility. A model that incorporates rotational hardening will be discussed on another occasion.

\section{ACKNOWLEDGMENT}

This paper reports on research carried out in the Technical Committee for the Mechanisms of Earthquake-Induced Ground Deformations (Chairman Akira Asaoka), a committee for seismic response research operating under the Japanese Geotechnical Society.

\section{REFERENCES}

1) Asaoka, A., Nakano, M. and Noda, T. (2000): Superloading yield surface concert for highly structured soil behavior, Soils and Foundations, 40(2), 99-110.

2) Asaoka, A., Noda, T., Yamada, E., Kaneda, K. and Nakano, M. (2002): An elasto-plastic description of two distinct volume change mechanisms of soils, Soils and Foundations, 42(5), 47-57.

3) Banerjee S., Davis R.O. and Sribalaskandarajah K. (1992): Simple double-hardening model for geomaterials, J.Geotech.Engrg,118, 889-901.

4) Drucker, D.C. and Prager, W. (1952): Soil mechanics and plastic analysis for limit design, Quarterly of Applied Mathematics, 10(2), 157-165.

5) Drucker D.C., Gibson R.E. and Henkel D.J. (1957): Soil mechanics and work hardening theories of plasticity, ASCE, 122, 339-346.

6) Einav, I. and Puzrin, A.M. (2004): Pressure-dependent elasticity and energy conservation in elasto-plastic models for soils, ASCE Journal of Geotechnical and Geoenvironmental Engineering, 130(1), 81-92.

7) Green, A.E. and Naghdi, P.M. (1965): A general theory of an elastic-plastic continuum, Archive for Rational Mechanics and Analysis, 18, 251-281.

8) Hashiguchi,K. (1989) : Subloading surface model in unconventional plasticity, Int. Journal of Solids and Structures, 25, 917-945. 